\title{
EFECTO DE LA VELOCIDAD DE DEPOSICIÓN EN IMPRESIONES 3D SOBRE LAS PROPIEDADES MECANICAS DEL POLIACIDO LACTICO
}

\section{EFFECT OF THE DEPOSITION SPEED IN 3D PRINTING, ON THE MECHANICAL PROPERTIES OF THE LACTIC POLYACID}

\author{
MSc. Holger Cacua*, PhD César Peña* \\ PhD. Bladimir Ramon * \\ * Universidad de Pamplona, Facultad de Ingenierías y Arquitectura, Departamento de \\ Ingeniería Mecánica, Mecatrónica e Industrial. \\ Km 1 vía Bucaramanga, Pamplona, Norte de Santander, Colombia \\ E-mail: \{ holger.cacua, cesarapc, hbladimir \}@ unipamplona.edu.co.
}

\begin{abstract}
Resumen: la manufactura aditiva es una técnica en la cual se deposita el material capa por capa hasta obtener una pieza tridimensional con geometrías complejas en tiempos relativamente cortos y a bajo costo. Sin embargo, las propiedades mecánicas de las piezas se ven afectadas por los diferentes parámetros de fabricación como altura de capa, velocidad de impresión, temperatura de extrusión, entre otros, tanto así, que los fabricantes de filamentos recomiendan la configuración de parámetros más adecuada para obtener la mayor resistencia en las piezas. En este artículo se presentan los resultados experimentales de las propiedades mecánicas a tracción de probetas normalizadas fabricadas a partir de un polímero biodegradable de poliácido láctico. Se evaluaron configuraciones obtenidas a diferentes velocidades de extrusión: $20 \mathrm{~mm} / \mathrm{s}, 36 \mathrm{~mm} / \mathrm{s}, 60$ $\mathrm{mm} / \mathrm{s}$ y $90 \mathrm{~mm} / \mathrm{s}$, mediante la técnica del modelado por deposición fundida o 3D. De acuerdo a los valores obtenidos se observó que aumentando en un 50\% la velocidad de impresión el tiempo de fabricación disminuye 4,9 horas y la resistencia a la tracción de las probetas solo se ve afecta en el $4 \%$. Por tanto, se logra concluir que al incrementar la velocidad de los hilos extruidos por la técnica de impresión en 3D, se disminuyen los tiempos de fabricación sin afectar considerablemente las propiedades mecánicas.
\end{abstract}

Palabras clave: Velocidad de deposición, Propiedades mecánicas a tracción, Impresión 3D.

\begin{abstract}
The additive manufacturing is a technique in which the material is deposited layer by layer to obtain a three-dimensional piece with complex geometries in relatively short times and at low cost. However, the mechanical properties of the pieces were affected by the different manufacturing parameters such as layer height, printing speed, extrusion temperature, among others, so much so that filament manufacturers recommend the most appropriate parameter configuration for obtain the greatest resistance in the pieces. In this paper we present the experimental results of the tensile mechanical properties of standardized test pieces made from a biodegradable polyacid lactic polymer. Configurations obtained at different extrusion speeds were evaluated: $20 \mathrm{~mm} / \mathrm{s}, 36 \mathrm{~mm} /$ $\mathrm{s}, 60 \mathrm{~mm} / \mathrm{s}$ and $90 \mathrm{~mm} / \mathrm{s}$, by means of the molten deposition or 3D modeling technique. According to the values obtained, it was observed that by increasing the printing speed by $50 \%$, the manufacturing time decreases by 4.9 hours and the tensile strength of the
\end{abstract}


specimens is only affected by $4 \%$. Therefore, it is possible to conclude that by increasing the speed of the extruded threads by the 3D printing technique, the manufacturing times are reduced without considerably affecting the mechanical properties.

Keywords: Deposition speed, tensile mechanical properties, 3D printing.

\section{INTRODUCCIÓN}

El modelado por deposición fundida (FDM) es una técnica de fabricación aditiva, en la cual la materia prima es un filamento polimérico, este es arrastrado por engranajes a través de un elemento denominado hot-end. Posteriormente, el material es sometido a una temperatura tal, que pasa del estado sólido al estado viscoso, y en consecuencia es extruido para dar origen a una pieza tridimensional (Arbeiter et al., 2018).

Esta técnica por FDM, permite fundir el material capa por capa, hasta formar un objeto con geometrías complejas y a menor costo. Con el paso de los años, el FDM ha sido de gran interés por diferentes sectores industriales, debido al impacto en la cadena de suministro al disminuir los tiempos de producción, eliminar costos de transporte, tiempos de espera, entre otros. (Kayfi et al., 2015).

Una desventaja de este método de FDM, es la baja resistencia mecánica y anisotropía de las piezas. (Dizon et al., 2018, demostró que la resistencia depende de la dirección de deposición de los hilos. En otro estudio, (Cacua et al., 2018) analizaron los efectos de variar el ángulo de inclinación en ejes cilíndricos.

Otros trabajos relacionados en el área de estudio, evaluaron el efecto de los parámetros de impresión en las propiedades mecánicas y posteriormente determinaron, el tipo de patrón y densidad con el cual las piezas ofrecen mejor comportamiento a diferentes fuerzas de aplicación, (Fernandez et al., 2016), (Croccolo et al, 2013).

Por su gran versatilidad, esta técnica por FDM ha permitido avances importantes en diferentes áreas de la investigación. En el sector salud, se utilizó para la fabricación de prótesis (Borjas y Flores, 2016), mientras que en el campo de la biología se utilizó para obtener cráneos de roedores, para ser utilizados en prácticas de instalación de dispositivos intra craneales (Pohl et al., 2013). En esta misma disciplina, se construyó por impresión en $3 \mathrm{D}$, un dispositivo que permite registrar la actividad neuronal de los peces (Rogers et al.,
2017). En ingeniería el FDM se ha utilizado para la fabricación de partes de máquinas, incluso de partes para la elaboración de otras impresoras (Kun, 2016), (Acuna et al., 2015), (Rodriguez et al, 2016). Por otro lado, se ha investigado el uso de esta técnica en la industria alimenticia (Lanaro et al., 2017).

Para lograr mayor resistencia en las piezas, los fabricantes recomiendan una velocidad $y$ temperatura de extrusión. En el caso del poliácido láctico (PLA), es de $60 \mathrm{~mm} / \mathrm{s}$ y temperatura de $205^{\circ} \mathrm{C}$. Un estudio desarrollado por (Song et al., 2017) demostró que con velocidades mayores $60 \mathrm{~mm} / \mathrm{s}$, se generan poros internos en las piezas y en consecuencia hay una reducción de su resistencia.

La presente investigación tiene como finalidad determinar el efecto de la velocidad de deposición de los filamentos, en las propiedades mecánicas de probetas normalizadas de un plástico denominado poliácido láctico o PLA. De acuerdo con los resultados se concluye que es posible disminuir los tiempos de fabricación de una pieza por esta técnica, sin afectar de manera considerable el comportamiento a tracción de la misma.

\section{MATERIALES Y METODOS.}

\subsection{Materiales}

Para esta investigación el material utilizado es el poliácido láctico o PLA. Este polímero hace parte de la familia de poliéster y se obtiene a partir de recursos renovables como el maíz, la yuca y la caña de azúcar. Cuando se mezcla con polímeros naturales permite desarrollar materiales con mejores propiedades y resistencia al agua. Una de las ventajas más relevantes del PLA es el grado de biocompatibilidad con los tejidos del cuerpo humano (Arraiza, 2008) .

El PLA es un polímero versátil que tiene muchas aplicaciones, incluyéndose en la industria textil, en la industria médica y sobre todo en la del empaquetado. Dentro de la industria textil, son 
conocidas las aplicaciones del PLA para la creación de telas empleadas en la tapicería, la elaboración de trapos y la confección de toldos y cubiertas resistentes a la luz U.V

Las propiedades del PLA según el fabricante Ultimaker se observan en la (tabla 1).

\section{Tabla 1. Propiedades mecánicas del PLA}

\begin{tabular}{lcc}
\hline \multicolumn{2}{c}{ Propiedades mecánicas del PLA } \\
\hline Módulo de elasticidad & $3,3-3,6$ & $\mathrm{GPa}$ \\
Límite de elasticidad & $55-72$ & $\mathrm{MPa}$ \\
Fuerza de tensión & $47-70$ & $\mathrm{MPa}$ \\
Elongación & $2,5-6$ & $\%$ \\
& & tensión \\
Módulo de compresión & $3,3-3,5$ & $\mathrm{GPa}$ \\
Fuerza de compresión & $66-86,4$ & $\mathrm{MPa}$ \\
Fuerza de flexión & 85 & $\mathrm{MPa}$ \\
Módulo de flexión & $3,1-3,6$ & $\mathrm{GPa}$ \\
Módulo de cortante & $5,7-6,3$ & $\mathrm{GPa}$ \\
Hardness-vickers & $17-22$ & $\mathrm{HV}$ \\
\hline
\end{tabular}

Para que las piezas obtengan las mejores propiedades mecánicas los fabricantes recomiendan utilizar los parámetros de impresión que se observan en la (tabla 2).

Tabla 2. parámetros de impresión recomendados por el fabricante de PLA

\begin{tabular}{lcc}
\hline \multicolumn{1}{c}{ PARÁMETRO } & VALOR & UNIDAD \\
\hline $\begin{array}{l}\text { Temperatura de } \\
\text { extrusión }\end{array}$ & 205 & ${ }^{\circ} \mathrm{C}$ \\
$\begin{array}{l}\text { Temperatura de la } \\
\text { cama caliente }\end{array}$ & 70 & ${ }^{\circ} \mathrm{C}$ \\
$\begin{array}{l}\text { Altura de capa } \\
\text { Perímetros internos }\end{array}$ & 0,2 & $\mathrm{Mm}$ \\
$\begin{array}{l}\text { Perímetros externos } \\
\text { Tipo de patrón }\end{array}$ & 7 & $\mathrm{UNI}$ \\
$\begin{array}{l}\text { Densidad } \\
\text { Velocidad de } \\
\text { impresión }\end{array}$ & 15 & $\mathrm{UNI}$ \\
\hline
\end{tabular}

\subsection{Fabricación de probetas}

Las probetas se fabricaron mediante el método de modelado por deposición fundida (FDM), haciendo uso de una impresora de código abierto Prusa Hephestos, a la cual se le realizaron modificaciones tanto estructurales como electrónicas

En la (tabla 3) se especifican las condiciones de impresión que utiliza la máquina de modelado por deposición fundida.
Tabla 3. Especificaciones de la impresora 3D

\begin{tabular}{ll}
\hline \multicolumn{2}{c}{ Especificaciones de la impresora 3D } \\
\hline Velocidad e impresión & $100 \mathrm{~mm} / \mathrm{s}$ \\
Volumen de las piezas & $215 \mathrm{~mm}(\mathrm{X})$ x 210mm $(\mathrm{Y}) \mathrm{x}$ \\
& $180 \mathrm{~mm}(\mathrm{z})$ \\
Resolución & Alta 60 micras, Media 200 \\
& micras, Baja 100 micra \\
Material recomendado & Filamento PLA $1.75 \mathrm{~mm}$ \\
Boquilla del extrusor & $0.4 \mathrm{~mm}$ \\
Disipador extrusor & Aletas con ventilador \\
& axial \\
\hline
\end{tabular}

Dentro de las características más llamativas de la impresora 3D, se puede observar (figura 1) el uso de una estructura metálica unida mediante juntas permanentes con soldadura 6013, con la cual se logra mayor rigidez y se espera mejorar la velocidad de fabricación.

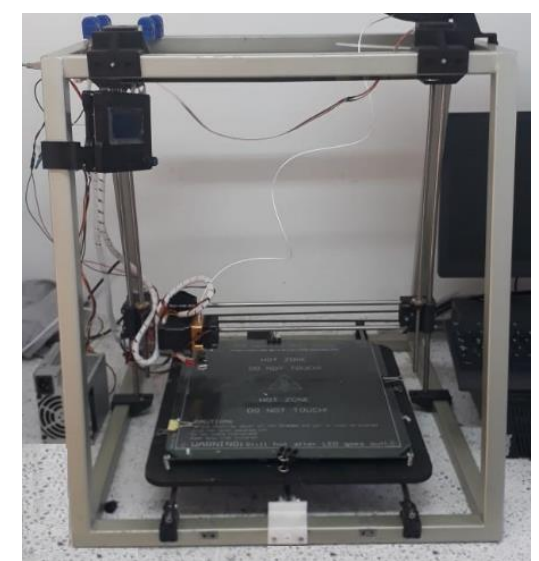

Fig. 1. impresora de código abierto Prusa Hephestos

\subsection{Ensayos de tracción.}

Los ensayos de tracción se realizaron en una máquina universal, Shimatzu de $600 \mathrm{KN}$ siguiendo los estándares de la norma ASTMA 638-10. La geometría de la probeta corresponde a una mancuerna y en algunos casos la referencian como hueso de perro. En la (figura 2) se observa la probeta utilizada en los ensayos de tracción. 


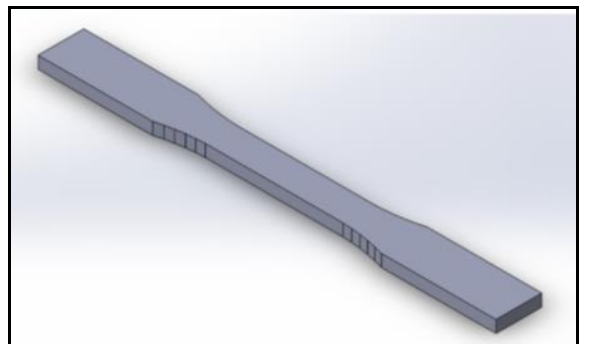

Fig. 2. Probeta tipo mancuerna para ensayos de tracción.

De igual forma, en la norma se establece las dimensiones de la probeta, para este caso se utilizaron las medidas que se observan en la (tabla 4), en donde se especifican la longitud calibrada y el espesor. Las otras dimensiones, la norma permite hacer modificaciones, que permiten adaptar la máquina para hacer el ensayo.

\section{Tabla 4. Dimensiones de la probeta para ensayos de tracción según norma 638-10}

\begin{tabular}{lc}
\hline \multicolumn{1}{c}{ MUESTRAS PARA ENSAYOS DE TRACCION } \\
\hline \multicolumn{1}{c}{ DIMENSION } & $\begin{array}{c}\text { Medida en } \\
(\mathbf{m m})\end{array}$ \\
Anchura W de sección estrecha. & 13 \\
L-Longitud de la sección estrecha & 57 \\
Anchura total WO. & 19 \\
LO-longitud total. & 165 \\
G-longitud calibrada & 50 \\
D-distancia entre las empuñaduras & 115 \\
R-Radio del filete & 76 \\
\hline
\end{tabular}

\section{RESULTADOS}

En la (tabla 5) se pueden apreciar los resultados de los ensayos de tracción realizados para las diferentes configuraciones, variando la velocidad de extrusión entre los sistemas estudiados.

Se puede observar que la velocidad más baja de 20 $\mathrm{mm} / \mathrm{s}$ se obtiene mayor resistencia a la tracción, esto se debe a que al aumentar la velocidad aumenta la vibración de la impresora, lo que en consecuencia puede conllevar a la formación de poros ocasionando que disminuya la adherencia entre las capas.

Tabla 5. Resultados ensayos de tracción.

\begin{tabular}{ccccc}
\hline $\begin{array}{c}\text { Velocidad } \\
(\mathbf{m m} / \mathbf{s})\end{array}$ & $\begin{array}{c}\text { Módulo } \\
(\mathbf{G P a})\end{array}$ & $\begin{array}{c}\text { Esfuerzo } \\
\text { Máximo } \\
(\mathbf{M P a})\end{array}$ & $\begin{array}{c}\text { Deformación } \\
(\mathbf{\%})\end{array}$ & $\begin{array}{c}\text { Tiempo de } \\
\text { fabricación } \\
\text { (Horas) }\end{array}$ \\
\hline $\mathbf{2 0}$ & 0,15 & 57,7 & 16,8 & 6 \\
$\mathbf{3 6}$ & 0,21 & 55,3 & 14,8 & 3 \\
$\mathbf{6 0}$ & 0,19 & 53,4 & 15,6 & 1,5 \\
$\mathbf{9 0}$ & 0,2 & 51,3 & 14,6 & 1,1 \\
\hline
\end{tabular}

El análisis de estos resultados demuestra que para el caso de la probeta fabricada con una velocidad de $90 \mathrm{~mm} / \mathrm{s}$ hay perdida en resistencia a la tracción de 6,4 MPa con respecto a la probeta fabricada con velocidad de $20 \mathrm{~mm} / \mathrm{s}$, pero se debe resaltar que la diferencia entre los tiempos de fabricación es bastante significaba puesto que con la velocidad de $20 \mathrm{~mm} / \mathrm{s}$ el tiempo de fabricación por probeta es de 6 horas mientras que con la velocidad de 90 $\mathrm{mm} / \mathrm{s}$ el tiempo es de 1,1 hora, es decir se logra un ahorro en el tiempo de fabricación 4,9 horas.

Se atribuye este logro a la rigidez estructural de la impresora 3D utilizada, puesto que fue fabricada con tubos metálicos unidos mediante juntas soldadas, las cuales no sufren desajuste por la vibración causada durante el funcionamiento, permitiendo utilizar velocidades de impresión más altas, en consecuencia, se logra fabricar piezas en tiempos menores sin afectar en gran medida su resistencia.

Con respecto a los valores arrojados con el porcentaje deformación, es evidente que el material disminuye la ductilidad en la medida que se incrementa la velocidad de deposición. Este fenómeno se puede analizar a partir de la pobre adhesión entre los filamentos al solidificarse, con lo cual se limitada sus fuerzas de unión entre las capas. Además, es posible la formación de concentradores de esfuerzos por el origen de poros internos al acelerar el proceso de deposición.

Es importante realizar un análisis de microscopia electrónica de barrido, que permita validar las hipótesis anteriormente mencionadas.

\section{CONCLUSIONES}

Los resultados obtenidos en los experimentos planteados permiten determinar que es posible aumentar la velocidad de impresión al emplear filamento de PLA de $1.75 \mathrm{~mm}$ a una temperatura de $205^{\circ} \mathrm{C}$ cuando se emplean estructuras robustas que disminuyen las vibraciones en las impresoras.

Para un aumento del 50\% de la velocidad recomendada inicialmente por el fabricante, se detectó tan solo una disminución del $4 \%$ de la resistencia a la tracción, lo cual es un logro significativo teniendo en cuenta que esto puede repercutir de forma positiva en la disminución de los tiempos de los procesos de producción y a su vez disminuir el consumo energético empleado teniendo en cuenta el menor uso de la cama caliente. 


\section{REFERENCIAS}

Acuna, F., Rivas, D., Chancusi, S., \& Navarrete, P. (2015). Design and Construction of a 3D Printer Auto Controller Wirelessly Through of Free Software. IEEE Latin America Transactions, 13(6), 1893-1898. https://doi.org/10.1109/TLA.2015.7164214

Alberto Lopez Arraiza. (2008). Correlaciones procesado-estructura-porpiedades en sitemas polimericos complejos basados en poliesteres biodegradables. evaluación de su posible uso en aplicaciones ecológicas y biomedicas (tesis de doctorado). Escuela Tecnica Superior de Ingenieria de Bilbao. Retrieved from

http://fondosdigitales.us.es/media/thesis/722/ S_TD_203.pdf

Alvernia Acevedo, S., \& Rico Bautista, D. (2017). Análisis de una red en un entorno IPV6: una mirada desde las intrusiones de red y el modelo TCP/IP. REVISTA COLOMBIANA DE TECNOLOGÍAS DE AVANZADA, 1(29).

Arbeiter, F., Spoerk, M., Wiener, J., Gosch, A., \& Pinter, G. (2018). Fracture mechanical characterization and lifetime estimation of near- homogeneous components produced by fused fi lament fabrication. ELSEVIER, 66(November 2017), 105-113.

https://doi.org/10.1016/j.polymertesting.2018 .01 .002

Borjas, R., \& Flores, W. (2016). Developing a human prosthesis using a 3D printer in Honduras. Proceedings of the 2015 IEEE 35th Central American and Panama Convention, CONCAPAN 2015, (Concapan $\mathrm{Xxxv).}$ https://doi.org/10.1109/CONCAPAN.2015.7 428465

Cacua, H., Peña, C., \& Ramon, B. (2018). Evaluation of the Mechanical Properties of Shafts Manufactured With 3D Printing for Different Inclinations of the Fused Deposition Layers. Indian Journal of Science and Technology, 11(September), 1-7. https://doi.org/10.17485/ijst/2018/v11i33/131 106

Croccolo, D., De Agostinis, M., \& Olmi, G. (2013). Experimental characterization and analytical modelling of the mechanical behaviour of fused deposition processed parts made of ABS-M30. Computational Materials Science, 79, 506-518. https://doi.org/10.1016/j.commatsci.2013.06. 041
Dizon, J. R. C., Espera, A. H., Chen, Q., \& Advincula, R. C. (2018). Mechanical characterization of 3D-printed polymers. Additive Manufacturing, 20, 44-67. https://doi.org/10.1016/j.addma.2017.12.002

Fernandez-Vicente, M., Calle, W., Ferrandiz, S., \& Conejero, A. (2016). Effect of Infill Parameters on Tensile Mechanical Behavior in Desktop 3D Printing. 3D Printing and Additive Manufacturing, 3(3), 183-192. https://doi.org/10.1089/3dp.2015.0036

J. Guzmán-Luna, I. D. Torres, J. F. Alvarez. (2014). Propuesta de un generador de aplicaciones educativas basadas en televisión digital usando arquitectura de cómputo en la nube. REVISTA COLOMBIANA DE TECNOLOGÍAS DE AVANZADA, ISSN: 1692-7257. 1(23).

J. Guzmán Luna, I. Torres and J. Alvarez. (2014). Propuesta de un generador de aplicaciones educativas basadas en televisión digital usando arquitectura de cómputo en la nube. REVISTA COLOMBIANA DE TECNOLOGÍAS DE AVANZADA, vol. 1, no. 23, 2014.

Kayfi, R., Ragab, D., \& Tutunji, T. a. (2015). Mechatronic System Design Project : A 3D Printer Case Study Mechatronic System Design Project: A 3D Printer Case Study. In IEEE jordan conference on Applied Electrical Engineering and Computing Technologies (AEECT) (pp. 1-6).

Kun, K. (2016). Reconstruction and development of a 3D printer using FDM technology. Procedia Engineering, 149(June), 203-211. https://doi.org/10.1016/j.proeng.2016.06.657

Lanaro, M., Forrestal, D. P., Scheurer, S., Slinger, D. J., Liao, S., Powell, S. K., \& Woodruff, M. A. (2017). 3D printing complex chocolate objects: Platform design, optimization and evaluation. Journal of Food Engineering, $215,13-22$. https://doi.org/10.1016/j.jfoodeng.2017.06.02 9

Pohl, B. M., Gasca, F., Christ, O., \& Hofmann, U. G. (2013). 3D printers may reduce animal numbers to train neuroengineering procedures. International IEEE/EMBS Conference on Neural Engineering, NER, 887-890. https://doi.org/10.1109/NER.2013.6696077

Rodriguez, E., Cortés, E., \& Peña, C. (2016). Application of the Qfd Methodology in the Development of. Revista Colombiana de Tecnologías de Avanzada. Retrieved from Results for \%22APLICACIÓN DE LA 
METODOLOGÍA QFD EN EL

DESARROLLO DE UNA IMPRESORA 3D

APPLICATION OF THE QFD

METHODOLOGY IN THE

DEVELOPMENT OF A 3D PRINTER\%22

in \%22All Documents\%22

Rogers, L. S., Van Wert, J. C., \& Mensinger, A. F. (2017). An implantable two axis micromanipulator made with a $3 \mathrm{D}$ printer for recording neural activity in free-swimming fish. Journal of Neuroscience Methods, 288, 29-33.

https://doi.org/10.1016/j.jneumeth.2017.06.0 12

S. A. A. Acevedo, D. R. Bautista. (2017). Análisis de una red en un entorno IPV6: una mirada desde las intrusiones de red y el modelo TCP/IP. REVISTA COLOMBIANA DE TECNOLOGÍAS DE AVANZADA, ISSN: 1692-7257. 1(29).

Song, Y., Li, Y., Song, W., Yee, K., Lee, K., \& Tagarielli, V. L. (2017). Measurements of the mechanical response of unidirectional 3Dprinted PLA, 123, 154-164.

https://doi.org/10.1016/j.matdes.2017.03.051 